\title{
Effects of Rubber Shock Absorber on the Flywheel Micro Vibration in the Satellite Imaging System
}

\author{
Changcheng $\mathrm{DENG}^{1,2}$, Deqiang $\mathrm{MU}^{1,3}$, Xuezhi JIA ${ }^{1}$, and Zongxuan $\mathrm{LI}^{1}$ \\ ${ }^{1}$ National \& Local United Engineering Research Center of Small Satellite Technology, Changchun Institute of Optics, \\ Fine Mechanics, and Physics ,Chinese Academy of Sciences, Changchun, 130033, China \\ ${ }^{2}$ University of Chinese Academy of Sciences, Beijing, 100039, China \\ ${ }^{3}$ Changchun University of Technology, Changchun, 130012, China \\ *Corresponding author: Changcheng DENGＥ-mail: changcheng0211@163.com
}

\begin{abstract}
When a satellite is in orbit, its flywheel will generate micro vibration and affect the imaging quality of the camera. In order to reduce this effect, a rubber shock absorber is used, and a numerical model and an experimental setup are developed to investigate its effect on the micro vibration in the study. An integrated model is developed for the system, and a ray tracing method is used in the modeling. The spot coordinates and displacements of the image plane are obtained, and the modulate transfer function (MTF) of the system is calculated. A satellite including a rubber shock absorber is designed, and the experiments are carried out. Both simulation and experiments results show that the MTF increases almost $10 \%$, suggesting the rubber shock absorber is useful to decrease the flywheel vibration.
\end{abstract}

Keywords: Micro vibration; flywheel; rubber shock absorber; integrated modeling; MTF; isolation experiment

Citation: Changcheng DENG, Deqiang MU, Xuezhi JIA, and Zongxuan LI, "Effects of Rubber Shock Absorber on the Flywheel Micro Vibration in the Satellite Imaging System," Photonic Sensors, 2016, 6(4): 372-384.

\section{Introduction}

During photographing of the in-orbit satellite, all kinds of motion of the moving components of the satellite, such as the rotation of the reaction flywheel, the jet of the propulsor, and the adjustment of solar panels, will cause the jitter response of the camera, thus affecting the image quality. The jitter response, also called the micro vibration system, cannot be measured and controlled by the attitude control system [1]. The rotation of the flywheel will have a relatively large impact on the image quality and is a main issue on the satellite imaging system [2].

To reduce the influence of micro vibration on imaging, different types of vibration control technologies are used [3], namely as passive and active vibration isolations, vibration absorption, vibration resistance, and dynamic designs. Among them, the vibration isolation method is most widely used. The principle of the vibration isolation method is adding a resilient liner (such as spring, rubber mats, and blankets) between the object and the supporting surface to isolate the vibration [4-6].

A rubber shock absorber [7] has the advantages of compact structure, low cost, good craftwork, etc., which is a very common passive vibration isolator and often used in many fields such as ships, buildings, and bridges. It is also easy to fabricate and meet the required stiffness and strength. Its

Received: 24 May 2016 / Revised: 16 June 2016

(C) The Author(s) 2016. This article is published with open access at Springerlink.com

DOI: $10.1007 / \mathrm{s} 13320-016-0349-1$

Article type: Regular 
damping ratio is $0.06-0.1$ and can absorb mechanical energy, especially high frequency mechanical energy. It can be bonded with metal, forming a multilayered structure to bear load, reduce system stiffness, and change its frequency range. A review on the application of the rubber shock absorber in aerospace can be found in [8]. An accurate and efficient finite element modeling [9] and static characteristics analysis of the rubber shock absorber was proposed by Haiting [12]. Sjoberg investigated rubber shock absorber dynamic modeling and dynamic characteristics [13]. Sjoberg used the method of fractional derivative to establish a simulation model [14]. In this study, a rubber shock absorber is added in the flywheel system to reduce the micro vibration of a satellite imaging system.

The principle of the integrated modeling is to integrate the analysis of the overall system, containing realistic structures, disturbance, optics and controls models, and their mutual interactions [15-17]. It is convenient for electronic transfer of data among all types of analysis, and this method can save operating time, heighten analyzing qualities, and achieve a dynamic front-to-end analysis of the disturbance to performance paths within the satellite. Compared with the traditional design and assessment methods that focus on single machine or single discipline or sub system level, integrated modeling can provide a systematic and comprehensive performance evaluation and error analysis, and guide systemic design. The ray tracing is used to calculate the location and direction of the output ray, and the angle between the ray and the optical axis. The calculation is based on the reflection lens displacement and the incident light, according to the refraction law. In this paper, the ray tracing method is used in the integrated modeling to analyze the influence of micro vibration on the satellite image.

An experiment is designed to use real products to verify the influence rubber shock absorber on the micro vibration of the flywheel in the satellite imaging system [18]. The data of vibration are obtained by the ground image acquisition and processing system, and the corresponding performance values are calculated. The results show that the rubber shock absorber can reduce the vibration in the transfer path and give out better image, which provides a guideline for the satellite design.

\section{Integrated modeling of the satellite}

The integrated modeling of the satellite consists of the modeling of the flywheel characteristic of vibration, the finite element modeling of the entire satellite platform, and the optical modeling based on ray tracing [19]. The process is shown in Fig. 1.

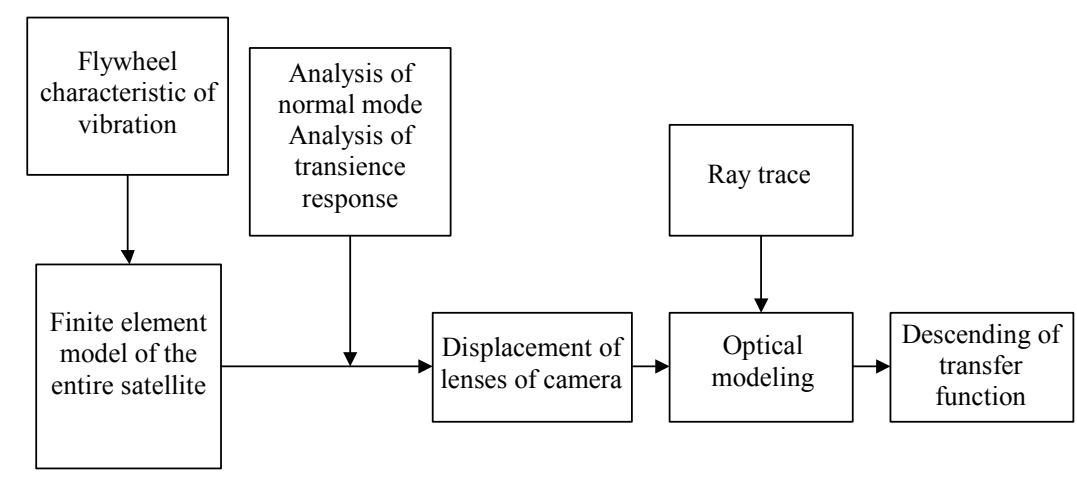

Fig. 1 Schematic of the integrated modeling. 


\subsection{Modeling of flywheel characteristic of vibration}

The micro vibration of the flywheel is caused by static unbalance and dynamic unbalance of the flywheel [20], as shown in Fig. 2. The static unbalance is caused by the deviation of the center of mass of the flywheel rotor from its rotating axis, and the flywheel can be considered as a rotor with two parts, the strict rotational symmetry part and a mass point $m_{s}$ part at a distance of $r_{s}$ from the flywheel shaft line. $F_{r}$ is the rotor inertia force. Dynamic unbalance refers to the uneven distribution of rotor mass which causes the cross product of inertia not zero. The rotor can be considered as two parts, a strict symmetry section part and 2 mass points $m_{d}$ part at a distance of $h$ in the axial direction. The mass point is at a distance $r_{d}$ from the rotating axis.

When the rotor rotates, the mass point $m_{s}$ in static imbalance is subjected to the radial force:

$$
F_{r}=U_{s} \omega^{2}
$$

where $U_{s}=m_{s} r_{s}$ is a flywheel mass property, and $\omega$ is the angular velocity.

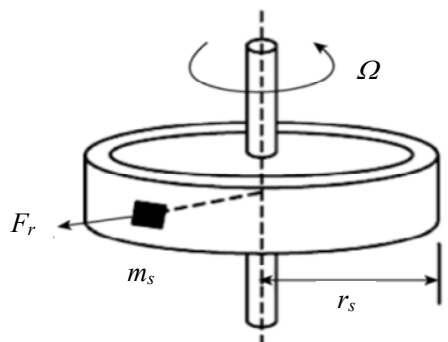

(a)

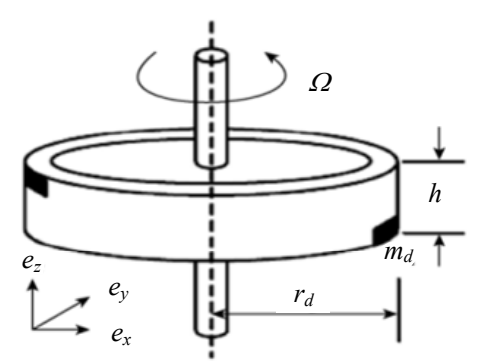

(b)

Fig. 2 Schematic of two types of the unbalanced flywheel [17]: (a) static unbalanced flywheel and (b) dynamic unbalanced flywheel.
For the dynamic unbalanced flywheel, the force caused by the moment of two mass points $m_{d}$ is

$$
T=U_{d} \omega^{2}
$$

where $U_{d}=m_{d} r_{d} h$ is a dynamic unbalance property.

\subsection{Finite element model of the entire satellite}

The finite element structure model is the basis of dynamic analysis (including the following modal analysis and transient analysis). The dynamics of a multi-degree-of-freedom system (structure) are described in the time domain by the equation [21]:

$$
\mathbf{M} \ddot{x}+\mathbf{C} \dot{x}+\mathbf{K} x=\mathbf{F}
$$

where $\mathbf{M}, \mathbf{C}$, and $\mathbf{K}$ are $N \times N$ dimensional matrices, of which $\mathbf{M}$ is the mass matrix, $\mathbf{C}$ is the damping matrix, and $\mathbf{K}$ is the stiffness matrix; $\mathbf{F}$ is the force matrix; $x$ is the displacement response. Introducing physical modal transformation $x=\boldsymbol{\Phi} q$ ( $\boldsymbol{\Phi}$ is the $N \times N$ matrix of the modal shape, $q$ is for the modal coordinates). Equation (3) can be decoupled as the modal space equation:

$$
\ddot{q}+2 \mathbf{Z} \boldsymbol{\Omega} \dot{q}+\mathbf{\Omega}^{2} q=\boldsymbol{\Phi}^{T} \mathbf{F} .
$$

Supposing retained $r$ modes (three rigid translational modes are not considered), $q \in \mathbf{R}^{\mathbf{r}}$ is modal coordinates; $\mathbf{Z} \in \mathbf{R}^{\text {rxr }}$ is the diagonal damping matrix; $\boldsymbol{\Phi} \in \mathbf{R}^{\mathrm{nxr}}$ is the mass normalized modal matrix; $\boldsymbol{\Omega} \in \mathbf{R}^{\text {rxr }}$ is the natural frequency of the diagonal matrix. Equation (5) is the transformed equation to state-space equation of (4):

$$
\left[\begin{array}{c}
\dot{x}_{p} \\
z \\
y
\end{array}\right]=\left[\begin{array}{lll}
\mathbf{A}_{p} & \mathbf{B}_{w} & \mathbf{B}_{u} \\
\mathbf{C}_{z} & \mathbf{D}_{z w} & \mathbf{D}_{z u} \\
\mathbf{C}_{y} & \mathbf{D}_{y w} & \mathbf{D}_{y u}
\end{array}\right]\left[\begin{array}{c}
x_{p} \\
w \\
u
\end{array}\right]
$$

where $x_{p}=[\mathbf{q}, \dot{\mathbf{q}}]^{T}, w, u \subset \mathbf{F}, w \in \mathbf{R}^{\mathbf{n}_{\mathbf{w}}}, u \in \mathbf{R}^{\mathbf{n}}, w$ is the disturbance input, and $u$ is the control input; $z \in \mathbf{R}^{\mathbf{n}_{2}}$ is the response output of the satellite; $y \in \mathbf{R}^{\mathbf{n}_{y}}$ is the control measure output. The system matrix is

$$
\left[\begin{array}{lll}
\mathbf{A}_{p} & \mathbf{B}_{w} & \mathbf{B}_{u} \\
\mathbf{C}_{z} & \mathbf{D}_{z w} & \mathbf{D}_{z u} \\
\mathbf{C}_{y} & \mathbf{D}_{y w} & \mathbf{D}_{y u}
\end{array}\right]=\left[\begin{array}{cccc}
0 & \mathbf{I} & 0 & 0 \\
-\boldsymbol{\Omega}^{2} & -2 \mathbf{Z} & \boldsymbol{\Phi}^{T} \boldsymbol{\beta}_{w} & \boldsymbol{\Phi}^{T} \boldsymbol{\beta}_{u} \\
\boldsymbol{\Phi} \boldsymbol{\beta}_{z} & 0 & 0 \\
\boldsymbol{\Phi} \boldsymbol{\beta}_{y} & 0 & 0
\end{array}\right] \text { (6) }
$$


where $\boldsymbol{\beta}_{w}, \boldsymbol{\beta}_{u}, \boldsymbol{\beta}_{z}$, and $\boldsymbol{\beta}_{y}$ are the modal selection matrices.

Parameters of the camera in the modeling are: focus $f=8 \mathrm{~mm}$; $F \#$ (F-number) $=13.3$; wavelength $500 \mathrm{~nm}-800 \mathrm{~nm}$. The finite element modeling of the satellite is completed by Patran/Nastran MSC software. The satellite model has 33893 nodes and 21856 elements. The model is used for the following simulation analysis to obtain displacements of all lenses. There is no displacement constrain in the model.

\subsection{Ray tracing}

The positions of the misaligned lenses are obtained after the vibration, and an optical path transmission is modeled with the ray tracing method in the study. By using kinematic concepts, the position of each lens is described with two coordinate systems, which are the lens coordinate system $o_{m} x_{m} y_{m} z_{m}$ and the implicated coordinate system oxyz. When the optical system is not subject to vibration, the lens coordinate system and implicated coordinate system are identical, and this is in the ideal position. When the vibration occurs, the lens coordinate system diverts from the implicated coordinate system. The lens coordinate system follows the lens, and the implicated coordinate system follows the base. Supposing the light transfers in reflection and the misaligned lens is parabolic, the misaligned lens is explained by an equation of lens in the lens coordinate, which is

$$
2 p z_{m}=x_{m}^{2}+y_{m}^{2} .
$$

The optical path transmission is based on the implicated coordinate. The equation of the misaligned lens relative to the implicated coordinate system is obtained by twice rotation and once translation of coordinates. Two points are taken from the center of up and low surfaces as shown in Fig. 3, i.e., Points 1 and 2, so the connection line of Points 1 and 2 is the optical axis, which can describe the displacement of the lens. Point 1 is the origin of the lens coordinate system, and the connecting direction of the two points is the $z_{m}$ direction of the coordinate axis. When Point 1 and the connecting direction of the line are determined, the position of the misaligned lens can be obtained. Because a parabolic surface is rotated, there is no need to consider the rotation of the lens.

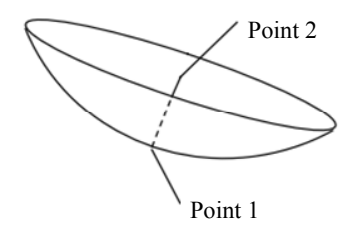

Fig. 3 Key points of lens.

The equation of the misaligned lens can be calculated as in [22]:

$$
\begin{aligned}
& F(x, y, z)=2 p\left(\left(x-\Delta x_{1}\right) \sin \beta+\cos \beta\left(\left(z-\Delta z_{1}\right) \cos \alpha+\right.\right. \\
& \left.\left.\left(y-\Delta y_{1}\right) \sin \alpha\right)\right)-\left(\left(x-\Delta x_{1}\right) \cos \beta-\sin \beta\left(\left(z-\Delta z_{1}\right) \cos \alpha+\right.\right. \\
& \left.\left.\left(y-\Delta y_{1}\right) \sin \alpha\right)\right)^{2}-\left(\left(y-\Delta y_{1}\right) \cos \alpha-\left(z-\Delta z_{1}\right) \sin \alpha\right)^{2} \\
& =0
\end{aligned}
$$

where $\alpha$ is the angle rotated around axis $x$ of coordinate $o x y z$, to receive coordinate $o x^{\prime} y^{\prime} z$; $\beta$ is the angle rotated around axis $y^{\prime}$ of coordinate $o x^{\prime} y^{\prime} z$ ', to receive coordinate $o x " y{ }^{\prime \prime} z$,' as shown in Fig. 4; $\left(\Delta x_{1}, \Delta y_{1}, \Delta z_{1}\right)$ is the misaligned displacement of Point $1 ; \quad\left(\Delta x_{2}, \quad \Delta y_{2}, \quad \Delta z_{2}\right)$ is the misaligned displacement of Point 2 . Assuming

$$
\left\{\begin{array}{l}
a=\Delta x_{2}-\Delta x_{1} \\
b=\Delta y_{2}-\Delta x_{1} \\
c=\Delta z_{2}-\Delta x_{1}
\end{array}\right.
$$

then

$$
\begin{aligned}
& \sin \alpha=\frac{b}{\sqrt{b^{2}+c^{2}}} \\
& \sin \beta=\frac{a}{\sqrt{a^{2}+b^{2}+c^{2}}} .
\end{aligned}
$$




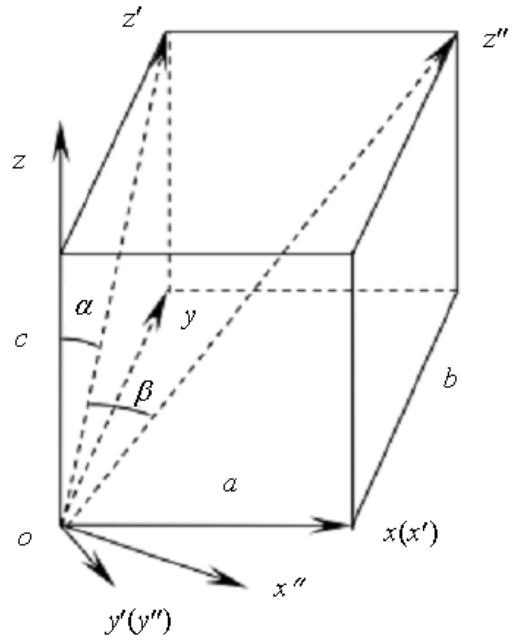

Fig. 4 Rotation of the coordinate system.

The ray tracing method for a single misaligned reflector application is shown in Fig. 5. When the displacements of Points 1 and 2 and the incident ray equation are given, the Matlab solving function is used to obtain the intersection between the misaligned lens and the incident ray. The vector in the direction of the light reflection is calculated by the vector reflection law, thus the angle between the reflection light and the optical axis is obtained.

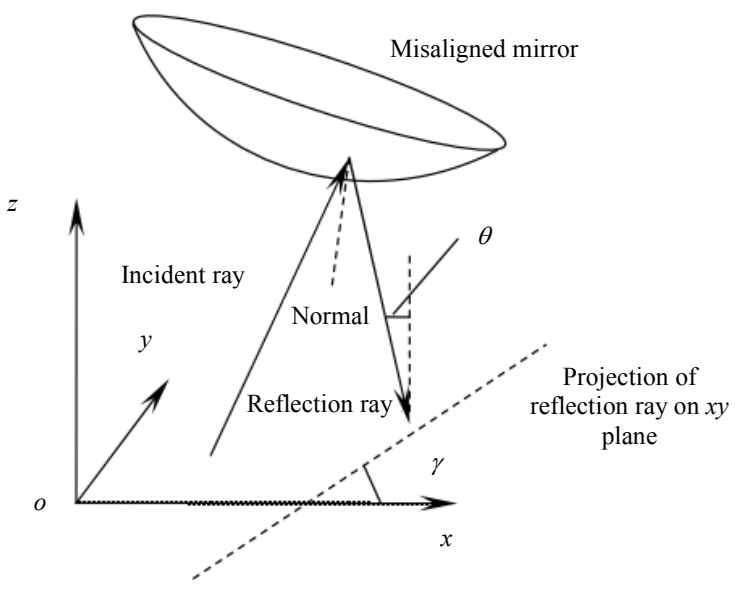

Fig. 5 Ray reflected by the misaligned lens.

The procedures of the ray tracing method for modeling a single misaligned lens optical transmission path are as follows:

(1) Obtain the equation of the misaligned lens by the coordinate transform $F(x, y, z)=0$.

(2) Calculate the intersection between the incident ray and misaligned lens, and the equation of the incident ray is

$$
\frac{x-x_{c-1}}{I_{x}}=\frac{y-y_{c-1}}{I_{y}}=\frac{z-z_{c-1}}{I_{z}}
$$

where $I=\left\{I_{x}, I_{y}, I_{z}\right\}$ is the vector of the incident ray.

(3) Obtain the normal vector $\mathbf{N}=\left\{\frac{\partial \mathbf{F}}{\partial x}, \frac{\partial \mathbf{F}}{\partial x}, \frac{\partial \mathbf{F}}{\partial z}\right\}$ of the intersection $\left(x_{c}, y_{c}, z_{c}\right)$, and $\mathbf{N}$ is normalized by the equation $\mathbf{n}=\frac{\mathbf{N}}{|\mathbf{N}|}$.

(4) Calculate the direction vector $\mathbf{R}=\mathbf{I}-2 \mathbf{n}(\mathbf{n} \cdot \mathbf{I})$ [23] of the reflected ray by the reflection equation in vectors, where $\mathbf{R}=\left\{R_{x}, R_{y}, R_{z}\right\}$ is the vector of the reflected ray, $\mathbf{n}$ is the normalized vector of the lens at the intersection, and the equation of the reflected ray is

$$
\frac{x-x_{c}}{R_{x}}=\frac{y-y_{c}}{R_{y}}=\frac{z-z_{c}}{R_{z}} .
$$

(5) Obtain the angle between the reflected ray and optic axis, $\theta=\arccos \frac{\mathbf{R} \cdot \mathbf{n}_{z}}{|\mathbf{R}|\left|\mathbf{n}_{z}\right|}$, where $\mathbf{n}_{z}=$ $\{0,0,-1\}$.

Because the reflected light is downward, the angle between the vertical downward direction and reflected ray can be acquired. The reflected ray is projected to the $x y$ plane, and then the angle $\gamma$ between the projection line and $x$ axis is obtained. When the ray tracing method is applied to the plural misaligned lens, the above five steps will be applied to each misaligned lens in turn.

\section{Design of rubber shock absorber}

Only one flywheel is used in the study, which has a total mass of $3.585 \mathrm{~kg}$ including four screws on the bracket (the mass of the bracket is $1.11 \mathrm{~kg}$ ). It is installed on the $y$ load plate, and each screw is mounted with a rubber shock absorber, as shown in Fig. 6.

The limit static stress $\sigma$ of the rubber shock absorber can be calculated as follows:

$$
\sigma=\frac{F^{\prime}}{S^{\prime}}
$$

where $F^{\prime}$ is the static load of the shock absorber; $S^{\prime}$ 
is the minimum bearing area of the shock absorber. For the low damping material, $\sigma$ is $1.8 \mathrm{MPa}$, thus the minimum area $S^{\prime}$ of the shock absorber is $6.39 \mathrm{~mm}^{2}$ for a static load of $11.5 \mathrm{~N}$. The screw is M5 then [24].

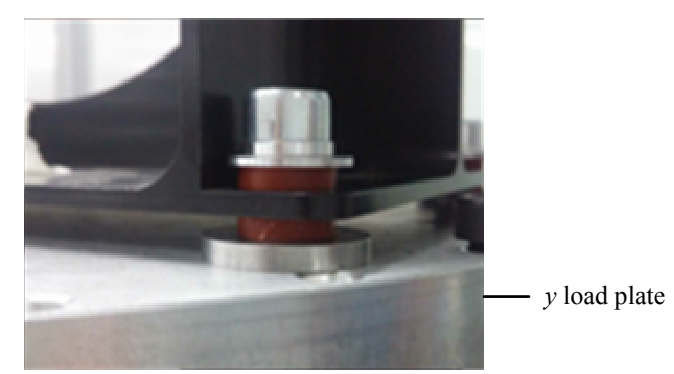

Fig. 6 Rubber shock absorber.

The natural frequency of the shock absorber $f_{n}$ is

$$
f_{n}=\frac{1}{2 \pi} \sqrt{\frac{K^{\prime} g}{G}}
$$

where $K^{\prime}$ is the dynamic stiffness of the shock absorber; $g$ is the acceleration of gravity; $G$ is the gravity of the shock absorber. According to technical indicators on the satellite equipment, the vibration frequency ranges are from $10 \mathrm{~Hz}$ to $2000 \mathrm{~Hz}$. If the vibration frequency meets $f>\sqrt{2} f_{n}$ (suppose its natural frequency $f_{n}=10 \mathrm{~Hz}$ ), rubber shock absorber damping takes effect, and the total dynamic stiffness calculated by (14) is $K^{\prime}=18516.33 \mathrm{~N} / \mathrm{m}$.

The complex shape of the shock absorber should be taken into account as a result of the parallel and series connection of some simple shapes. Thus the total rigidity of the connection is written as

$$
\begin{aligned}
& k_{c}=k_{c 1}+k_{c 2}+\cdots \\
& \frac{1}{k_{b}}=\frac{1}{k_{b 1}}+\frac{1}{k_{b 2}}+\cdots .
\end{aligned}
$$

For easy calculation, the stress-strain relationship of the rubber damper is linear, then

$$
K_{S}=\frac{S E_{c}}{h}
$$

where $K_{S}$ is the static stiffness of the shock absorber, and $\frac{K^{\prime}}{K_{S}}$ is generally $1.2-2 ; S$ is the effective load area of the shock absorber; $E_{c}$ is the effective compression modulus of shock absorber; $h$ is the height of the shock absorber.
The effective compression modulus can be expressed mathematically as

$$
E_{c}=E_{0}\left(1+2 K^{\prime \prime} C^{2}\right)
$$

where $E_{0}$ is the elastic modulus of rubber materials; $C$ is a shape factor of the rubber shock absorber, which is the ratio between the loaded area and not loaded area, and can be calculated by $C=\frac{D}{4 h^{\prime}}$, where $D$ is the diameter, and $h^{\prime}$ is the height of the shock absorber. To ensure the stability of the shock absorber, not bending, the condition $\frac{h^{\prime}}{D}<1$ is required; $K^{\prime \prime}$ is the correction factor of the material properties, which ranges from 0.5 to 1 .

Assuming $\frac{K^{\prime}}{K_{S}}=1.5$, then the total stiffness is $K_{S}=12344.22 \mathrm{~N} / \mathrm{m}$. Supposing four shock absorbers bear the load uniformly, the static stiffness of each shock absorber is $K_{S}^{\prime}=3086.05 \mathrm{~N} / \mathrm{m}$ according to (15); the effective compression modulus of each shock absorber is $E_{c}=0.163 \mathrm{MPa}$ according to (17); the elastic modulus of the damper material is $E_{0}=$ $108.67 \mathrm{kPa}$ according to (18) and the shock absorber structure size (as shown in Fig. 7). The elastic modulus of the rubber is about $100 \mathrm{kPa}$, so it can be used [25].

The rubber is made in 703 Institute, and the damping ratio of the rubber shock absorber is 0.1 . The dimension of the rubber shock absorber is used in the following simulation and experimental design.

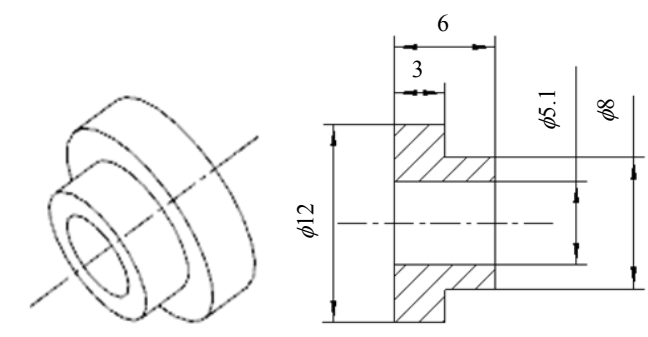

Fig. 7 Structure of the rubber shock absorber.

\section{Vibration simulation analysis}

\subsection{Modal analysis}

Modal analysis is to determine the dynamic characteristics of the camera, which provides given 
order natural frequencies and mode shapes, investigate the dynamic stiffness of the camera, and assess its other dynamic performances [26]. This is the basis of other dynamic response analyses (such as transient analysis). From the modal analysis, the weakness of structural rigidity is obtained which is used for the design optimization. The first few modals and shapes of the entire satellite are shown in Table 1. The modal is low, but it meets the design. The coordinate system is that the optical axis is $z$ axis, which consists of axes $x, y$, and $z$, and follows the right-handed coordinate system.
Table 1 Modal and shapes.

\begin{tabular}{ccc}
\hline Modal order & Frequency $(\mathrm{Hz})$ & Shapes \\
\hline 1 & 15.93 & Swinging about $y z$ plane \\
2 & 17.242 & Swinging about $x z$ plane \\
3 & 30.618 & Twisting about $z$ axis \\
5 & 35.694 & Swinging about $x y$ plane \\
\hline
\end{tabular}

\subsection{Transient analysis}

The static and dynamic unbalanced forces and moments are tested by the HR-FP3402 force platform as shown in Fig. 8(a). Figures 8 (b) and 8(c) show an example of the force results (at the speed of $3000 \mathrm{rpm}$ ) for the flywheel without and with isolation.

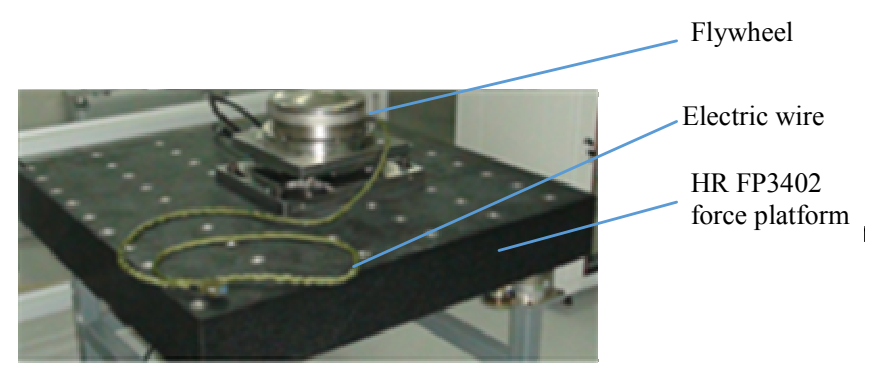

(a)

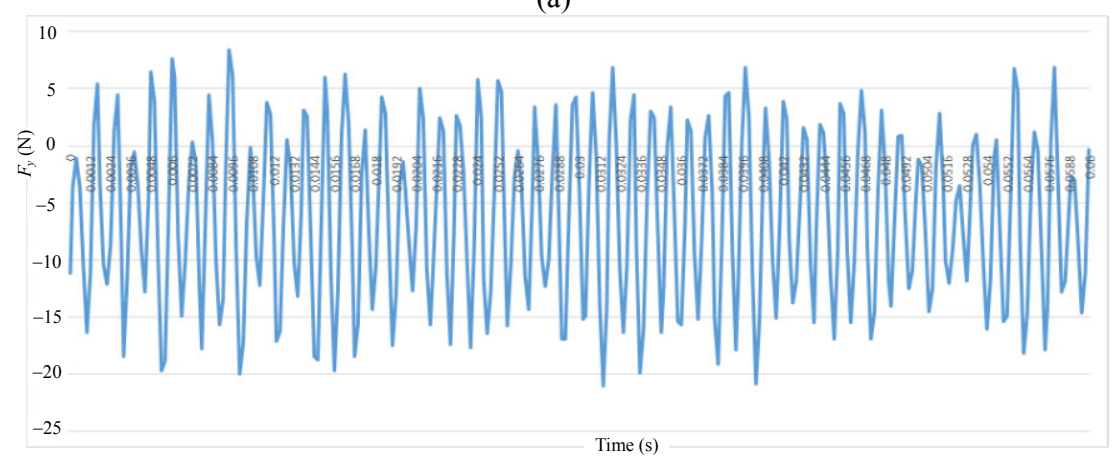

(b)

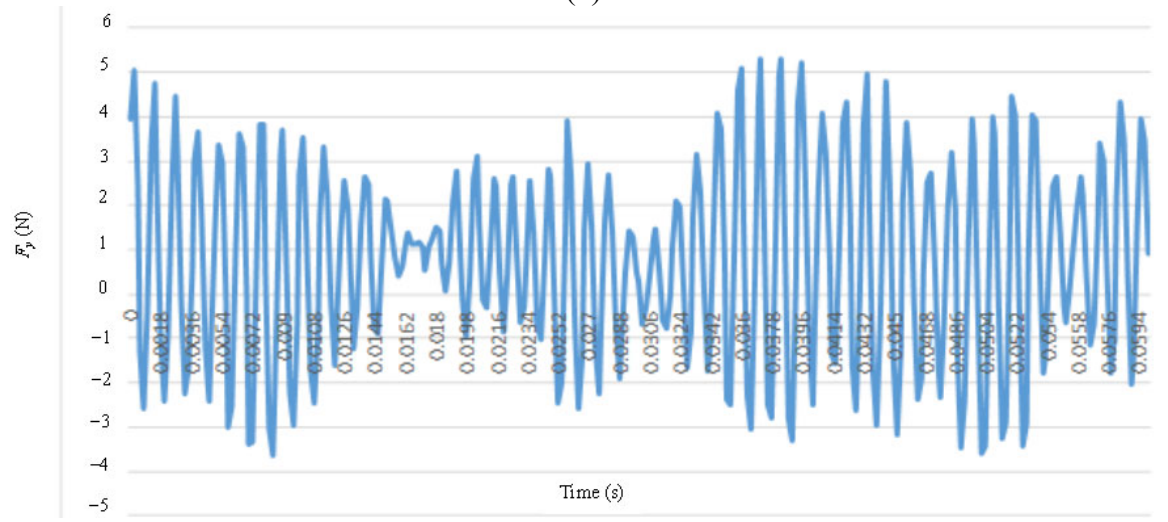

(c)

Fig. 8 Test platform and tested force results of the flywheel: (a) test platform for the force and moment of the flywheel, (b) test result $F_{y}$ of the flywheel without isolation, and (c) test result $F_{y}$ of the flywheel with isolation. 
The forces and moments of the flywheel at various speeds are tested. Only the disturbance data at $3000 \mathrm{rpm}$ are imported into Patran, because the flywheel runs mostly at this speed. The transient displacements of all the lenses and focus plan along the $x$ axis and $y$ axis are obtained by the Nastran calculation, as shown in Figs. 9 and 10. The meaning of the related nodes is shown in Table 2. The displacement data are the inputs of calculation of the modulate transfer function (MTF) in the next section.

Table 2 Meaning of the node.

\begin{tabular}{cc}
\hline Node & Lens \\
\hline 11658 & Second lens \\
12479 & Primary lens \\
12480 & Focal plane \\
12481 & Focusing lens \\
12482 & Folding lens \\
12483 & Third lens \\
\hline
\end{tabular}

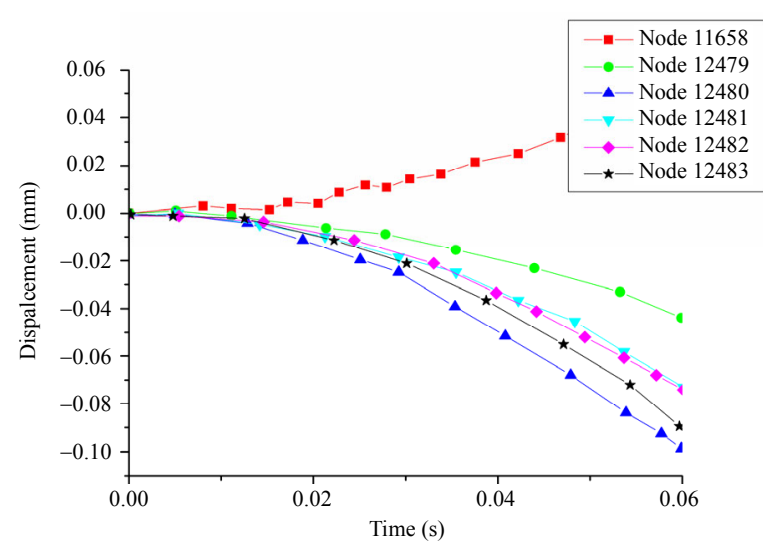

(a)

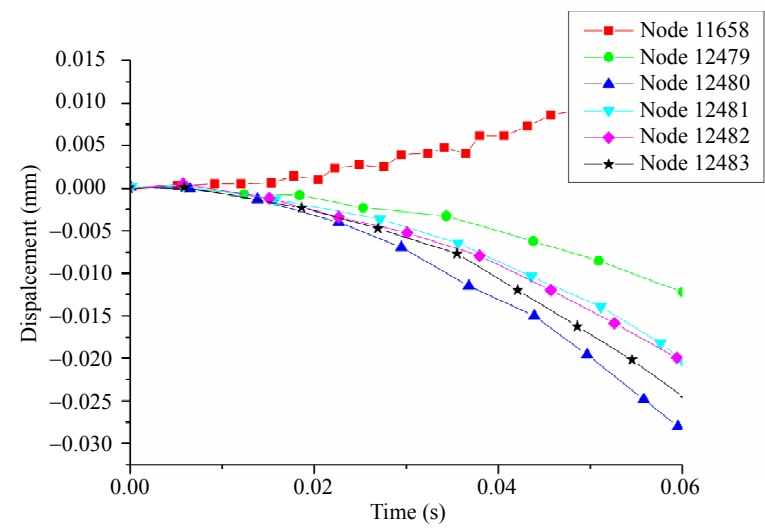

(b)

Fig. 9 Displacement in the $x$ axis: (a) without the rubber shock absorber and (b) with the rubber shock absorber.

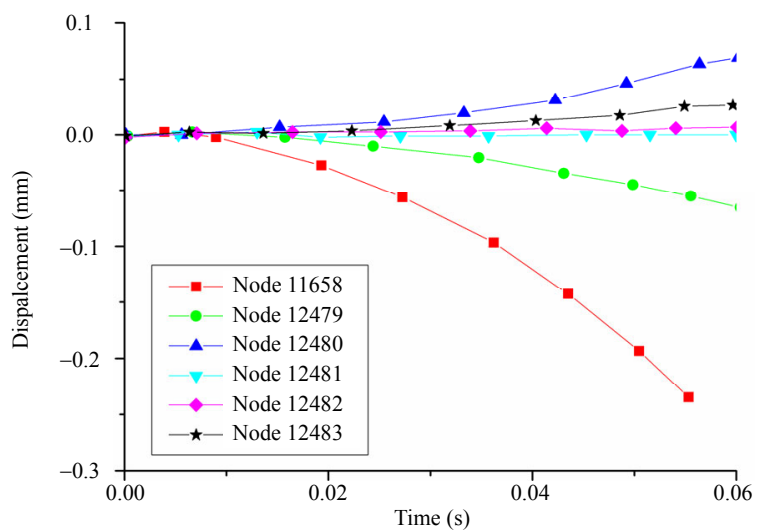

(a)

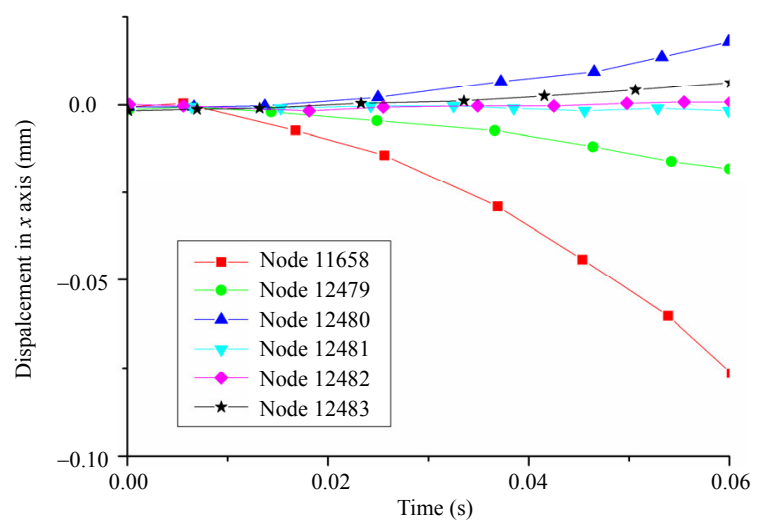

(b)

Fig. 10 Displacement in the $y$ axis: (a) without the rubber shock absorber and (b) with the rubber shock absorber.

\subsection{Calculation of MTF}

In exposure, displacements of the primary lens, second lens, third lens, folding lens, focusing lens, and the focal plane $x, y$ directions are imported into the Matlab program from Patran. Then each set of vibration data is sent to Zemax via the dynamic link. The vibration information about the displacements of the light spot in the image plane is obtained at the sampling time by ray tracing. By repeating each sampling data in integration time, the displacement of the light spot image at the image plane in the exposure can be obtained. The displacement may be interpreted as the image shift probability distribution of the sample generated by vibration. The imaging MTF of vibration is calculated with the optical transfer function (OTF) formula (19) and formula of $\mathrm{N}$-order statistical moments (20) [28], and the 
flowchart is shown in Fig. 11.

$$
\begin{gathered}
\operatorname{OTF}(\omega)=\sum_{n=0}^{\infty} \frac{m_{n}}{n !}(-\mathrm{j} \omega)^{n} \\
m_{n}^{x}=\frac{1}{S} \sum_{i=1}^{s} x_{i}^{n}
\end{gathered}
$$

where OTF is the optical transfer function; $\left\{x_{i}\right\}(i=1,2,3, \cdots, S)$ is the sampling sequence; $m_{n}^{x}$ is for the $N$-order statistical moments of movement.

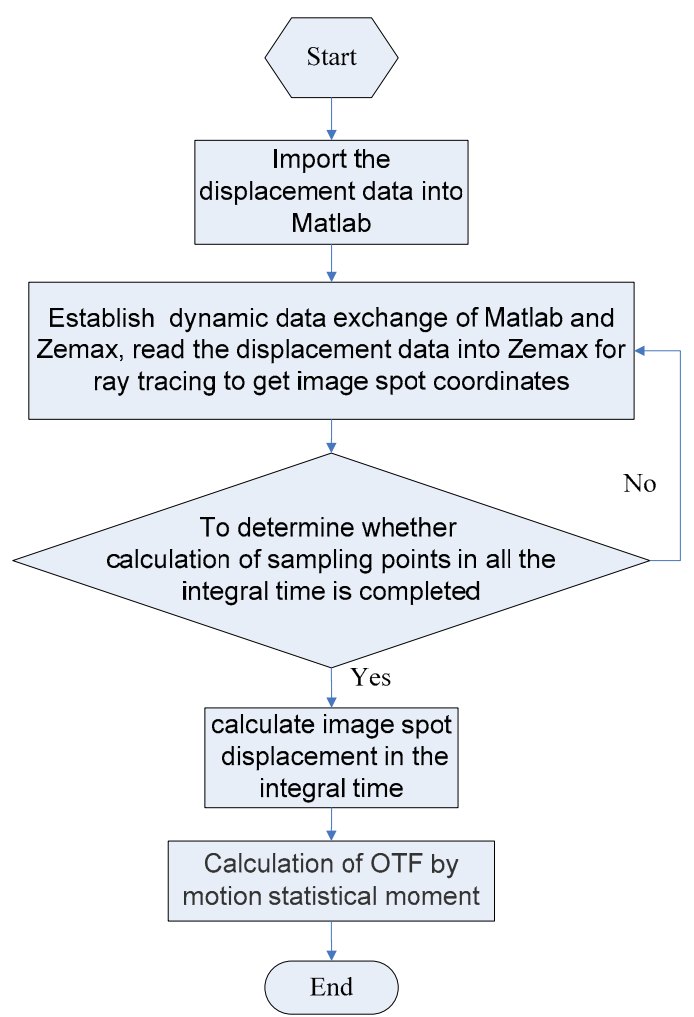

Fig. 11 Flowchart of MTF calculation.

The spot displacements in the focal plane in the $x$ axis and $y$ axis are shown in Fig. 12. The MTF is 1 in the ideal case without vibration. The calculated MTF without the rubber shock absorber at the Nyquist frequency $(571 \mathrm{p} / \mathrm{mm})$ is 0.88 along the $x$ axis and is 0.90 along the $y$ axis as shown in Fig. 13. Generally, the MTF of vibration can be considered as the average of two axes, i.e., 0.89. With the rubber shock absorber added, the MTF at the Nyquist frequency is 0.97 along the $x$ axis and is 0.99 along the $y$ axis, so the whole MTF of the system is 0.98 , increased by $10.1 \%$ compared with the system without the rubber shock absorber.

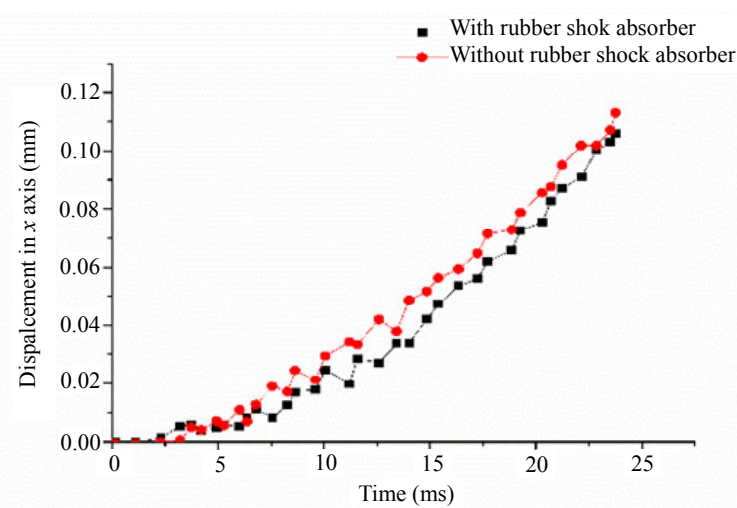

(a)

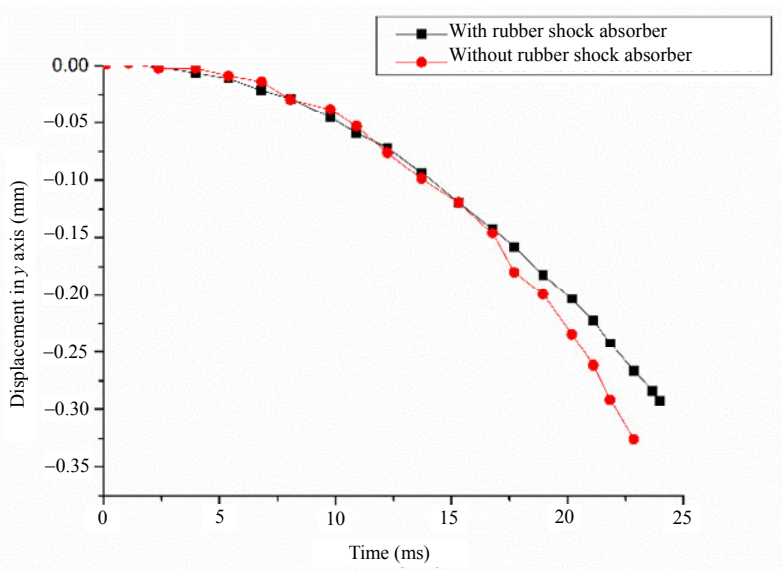

(b)

Fig. 12 Spot displacement in the focal plane: (a) in the $x$ axis and (b) in the $y$ axis.

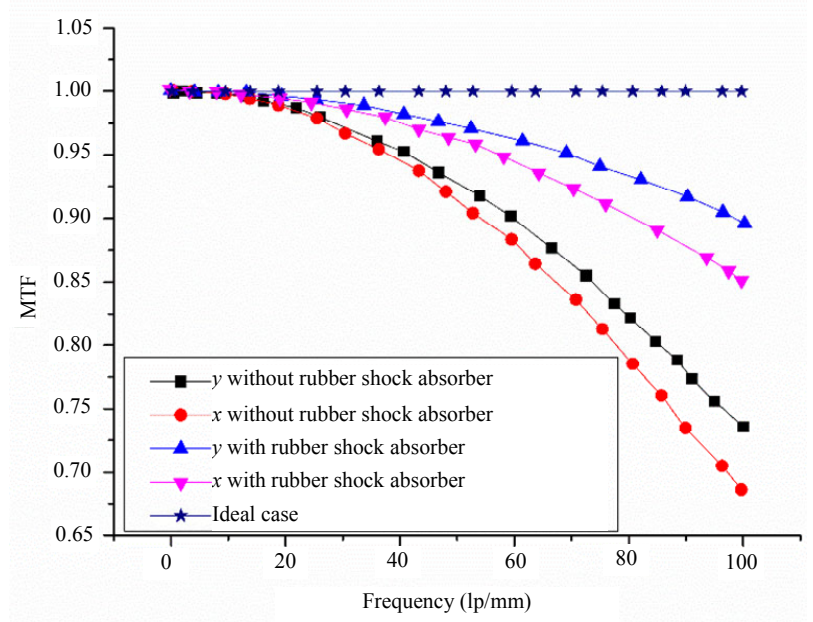

Fig. 13 Effects of vibration on the MTF.

\section{Experiment}

\subsection{Experimental system}

The principle of the experiment is plotted in 
Fig. 14. The experimental equipment consists of a satellite containing a camera, a rubber shock absorber, a reaction flywheel, a collimator, a crane, a set of charge coupled device (CCD) components, a calibration target, an alignment equipment, a data acquisition system, and a computer.

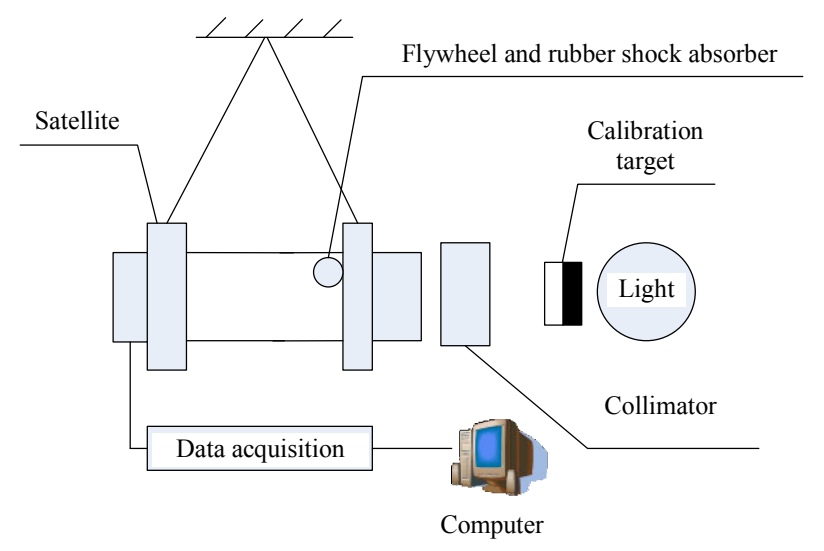

Fig. 14 Schematic of experimental system.

The flywheel rotating axis is vertical to the optic axis. The working conditions with the rubber shock absorber and without the rubber shock absorber were investigated. To intimate the real flying condition of satellite and the gravity environment in space, the satellite is suspended with a soft sling $[29,30]$.

Before experiment, we need to check that the bolts are screwed down and circuit is expedited. When the experiment starts, the flywheel is powered on, the speed of flywheel increases gradually from 0 to $3000 \mathrm{rpm}$, and the speed of the flywheel is controlled by the software platform. When the speed reaches $300 \mathrm{rpm}$, the CCD starts to sample, and then the speed increases by $200 \mathrm{rpm}$ by step.

A static target is illuminated by a light source, and the light passes through collimator and lenses of the camera. The light is focused on the focal plane, and the CCD sensor is used for imaging. When the flywheel speed is stable, the images are sampled at a corresponding speed. The data are acquired by the ground image acquisition and processing system, and the corresponding MTF is calculated. The data are analyzed and calculated in the time domain and frequency domain, outputing graphs in 2 dimensions, to describe the changes in related parameters of the vibration. In the process of camera imaging, the effect of the reaction flywheel on the imaging quality of the camera is observed by varying the reaction wheel speed.

Two coordinate systems are used in the experiments: the satellite and the flywheel coordinate systems. In the satellite coordinate system, the $z$ axis is the optic axis, the $x$ axis is the direction of flight, and the $y$ axis is decided following the right-handed coordinate system. In the flywheel coordinate system, the $z$ axis is the rotation axis, and $x$ and $y$ axes are decided by the right-handed coordinate system.

\subsection{Experimental result}

It is shown in Fig. 15 that the amplitude limit in the time domain is 0.4 pixel to -0.4 pixel $(1$ pixel $=$ 0.2 ) at the speed of $3000 \mathrm{rpm}$ without the rubber shock absorber, while the amplitude limit with the rubber shock absorber is 0.2 pixel to -0.2 pixel. The spectrum can be obtained by the Fourier transform of the results in Fig. 15 and is shown in Fig. 16. With the rubber shocker absorber added, the amplitude decreases in all the ranges of frequency, and the summit of amplitude decreases $87.5 \%$, from 0.2386 at $537.1 \mathrm{~Hz}$ to 0.02978 at $85.45 \mathrm{~Hz}$. Figure 17 shows the MTF at the Nyquist frequency as a function of the started phase. The MTF is varied at different starting phases, which is because the time of exposure is very short and the time of vibration is relatively long. It is also shown that the MTF with the rubber shock absorber is generally higher than that without the rubber shock absorber. At 0.5 (starting phase), the MTF increases from 0.8897 to 0.97 by adding the rubber shock absorber, increasing by $9 \%$. It can be compared with Fig. 14, providing the MTF is taken at the Nyquist frequency. The simulated and experimental results of the MTF with 
and without the rubber shock absorber are listed in Table 3, and both the simulation and experiment show the rubber shock absorber increases the MTF almost $10 \%$, and the experimental results matches well with the simulation values.

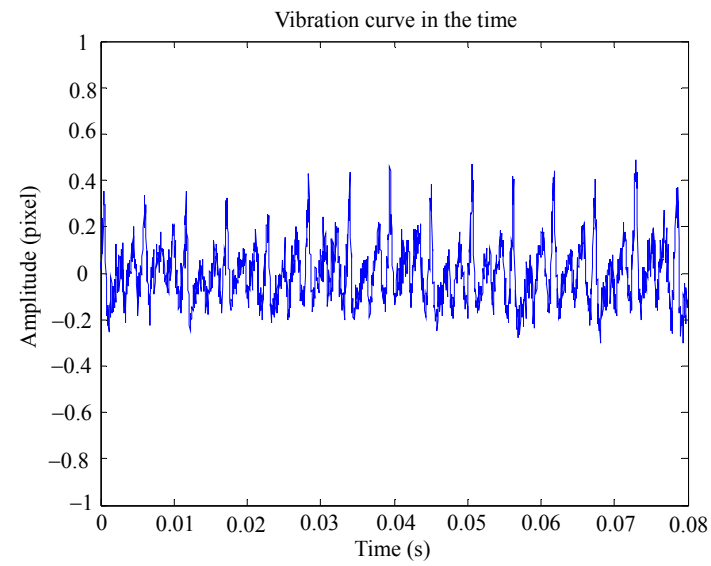

(a)

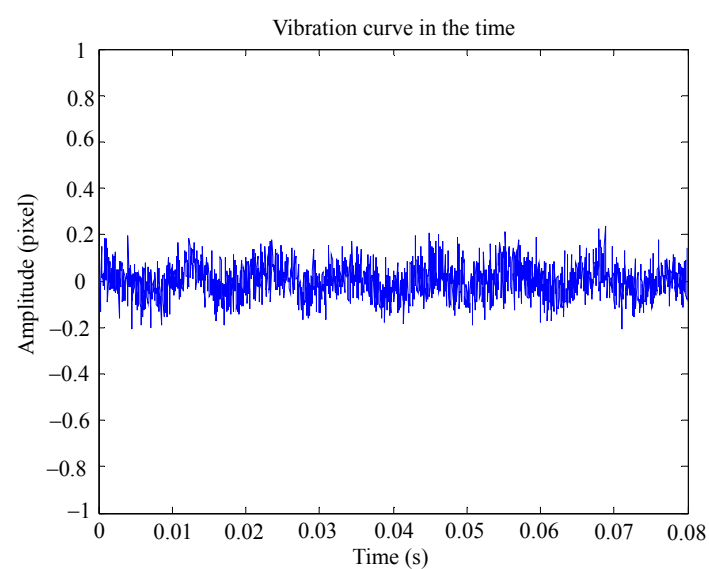

(b)

Fig. 15 Amplitude in the time domain: (a) without the rubber shock absorber and (b) with the rubber shock absorber.

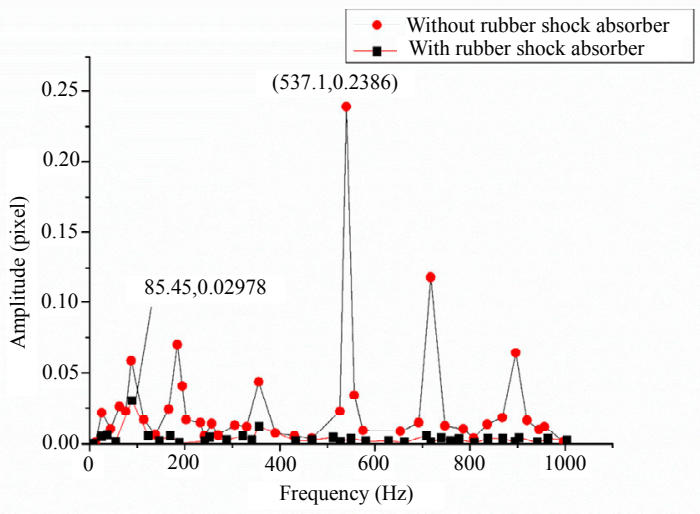

Fig. 16 Amplitude in frequency.

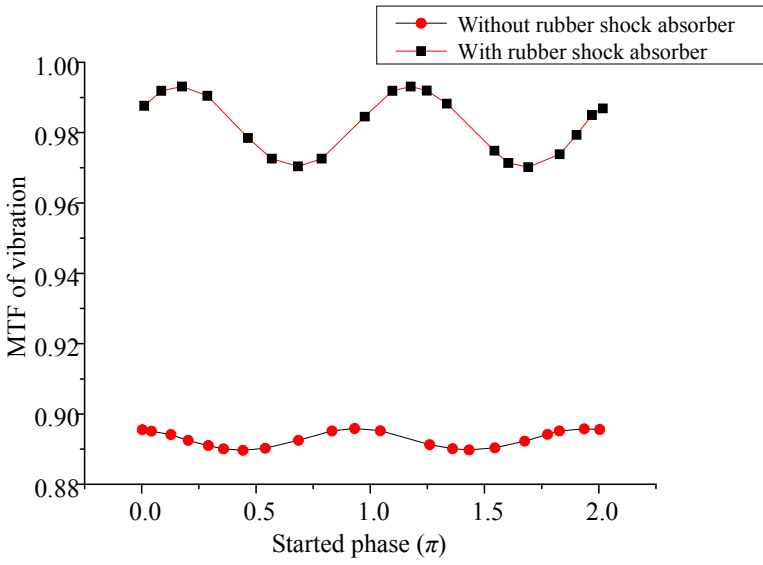

Fig. 17 MTF of vibration.

Table 3 MTF contrast

\begin{tabular}{ccc}
\hline Case & $\begin{array}{c}\text { MTF of simulation at } \\
\text { Nyquist frequency }\end{array}$ & $\begin{array}{c}\text { MTF of experiment at } \\
\text { Nyquist frequency }\end{array}$ \\
\hline $\begin{array}{c}\text { Without rubber shock } \\
\text { absorber }\end{array}$ & 0.89 & 0.8897 \\
$\begin{array}{c}\text { With rubber shock } \\
\text { absorber }\end{array}$ & 0.98 & 0.97 \\
\hline
\end{tabular}

\section{Conclusions}

To reduce the effect of micro vibration of the flywheel on the camera imaging system, a rubber shock absorber is used in the satellite system, and its effects are analyzed by the simulation and experimental method. In the simulation, an integrated modeling analysis based on ray tracing is developed. A real product is designed and tested. The experimental results match well with the simulated ones, and both show that the MTF increases almost $10 \%$, which confirms the rubber shock absorber is helping to decrease the effect of the flywheel vibration on the camera images.

\section{Acknowledgment}

The author would like to thank the great help from Changchun Iinstitute of Optics, Fine Mechanics, and Physics, Chinese Academy of Sciences during the experiment.

Open Access This article is distributed under the terms of the Creative Commons Attribution 4.0 International License (http://creativecommons.org/ licenses/by/4.0/), which permits unrestricted use, distribution, and reproduction in any medium, provided you give appropriate credit to the original author(s) and the source, 
provide a link to the Creative Commons license, and indicate if changes were made.

\section{References}

[1] Z. Wei, D. Li, Q. Luo, and J. Jiang, "Modeling and analysis of a flywheel microvibration isolation system for spacecrafts," Advances in Space Research, 2015, 55(2): 761-777.

[2] D. O. Lee, G. Park, and J. H. Han, "Experimental study on on-orbit and launch environment vibration isolation performance of a vibration isolator using bellows and viscous fluid," Aerospace Science and Technology, 2015, 45: 1-9.

[3] C. Liu, X. Jing, S. Daley, and F. Li, "Recent advances in micro-vibration isolation," Mechanical System and Signal Processing, 2015, 56(1): 55-80.

[4] V. G. Geethamma, R. Asaletha, N. Kalarikkal, and S. Thomas, "Vibration and sound damping in polymers," Resonance, 2014, 19(9): 821-833.

[5] M. Abdulhadi, "Stiffness and damping cofficients of rubber," Archive of Applied Mechanics, 1985, 55(6): 421-427.

[6] S. E. Klenke and T. J. Baca, "Structural dynamics test simulation and optimization for aerospace components," Expert Systems with Applications, 1996, 11(4): 82-89.

[7] J. C. Dixon, The shock absorber handbook. New York: SAE International, 2007.

[8] J. Njuguna and K. Pielichowski, "The role of advanced polymer materials in aerospace," Research Gate, 2013: 1-48.

[9] A. Dall'Asta and L. Ragni, "Nonlinear behavior of dynamic systems with high damping rubber devices," Engineering Structure, 2008, 30(12): 3610-3618.

[10] D. W. Nelson and N. W. Nelson, "Finite element analysis in design with rubber," Chemistry and Technology, 1990, 63(3): 368-406.

[11] T. J. R. Hughes, The finite element method: linear static and dynamic finite element analysis. New Jersey: Prentice Hall, 2000.

[12] L. Chen, "Numerical methods for analysing static characteristics of rubber isolator," Journal of Vibration and Shock, 2005, 25(123-124): 56-61.

[13] M. Sjoberg, "On dynamic properties of rubber isolators," Ph.D. dissertation, Kungliga Tekniska högskolan, 2002.

[14] M. Sjoberg, "Rubber isolators measurements and modelling using fractional derivatives and friction," SAE Technical Paper, 2000, 1(3518): 133-144.

[15] M. D. Lieber, "Space-based optical system performance evaluation with integrated modeling tools," SPIE, 2004, 5420: 85-96.

[16] D. M. LoBosco, C. Blaurock, S. J. Chung, and D. W. Miller, "Integrated modeling of optical performance for the Terrestrial Planet Finder structurally connected interferometer," SPIE, 2004, 5497: 278-289.

[17] O. L. D. Weck, D. W. Miller, G. J. Mallory, and G. E. Mosier, "Integrated modeling and dynamics simulation for the next generation space telescope (NGST)," SPIE, 2000, 4013: 920-934.

[18] W. Zhou and D. Li, "Experimental research on a vibration isolation platform for momentum wheel assembly," Journal of Sound and Vibration, 2013, 332(5): 1157-1171.

[19] D. W. Miller, O. L. D. Weck, and G. E. Mosier, "Framework for multidisciplinary integrated modeling and analysis of space telescope," Integrated Modeling of Telescopes, 2002, 4757: 1-18.

[20] L. M. Elias, F. G. Dekens, I. Basdogan, and L. A. Sievers, "Methodology for modeling the mechanical interaction between a reaction wheel and a flexible structure," SPIE, 2003, 4852: 541-555.

[21] D. O. Lee, J. S. Yoon, and J. H. Han, "Development of integrated simulation tool for jitter analysis," International Journal of Aeronautical and Space Sciences, 2012, 13(1): 64-73.

[22] A. S. Glassner, "An introduction to ray tracing," Morgan Kaufmann Publishers, 1989, 34(2): 417-417.

[23] M. Katz, Introduction to geometrical optics. New Jersey: World Scientific, 2002.

[24] H. T. Yang, J. Z. Cao, Z. Y. Fan, and W. N. Chen, "The research of the high precision universal stable reconnaissance platform in near space," International Symposium on Photoelectronic Detection and Imaging, 2011, 8196(3): 111-116.

[25] S. Hadden, T. Davis, P. Buchele, J. Boyd, and T. L. Hintz, "Heavy load vibration isolation system for airborne payloads," SPIE, 2001, 4332: 171-182.

[26] B. Zhang, X. Wang, and Y. Hu, "Integrated modeling and optical jitter analysis of a high resolution space camera," SPIE, 2012, 8415: 841508-1841508-7.

[27] O. Hadar and N. S. Kopeika, "Numerical calculation of MTF for image motion: experimental verification," SPIE, 1992, 1697: 183-197.

[28] O. Hadar, I. Dror, and N. S. Kopeika, "Real-time numerical calculation of optical transfer function for 
image motion and vibration. Part 1: experimental verification," Optical Engineering, 1997, 33(2): 566-578.

[29] W. Zhou, L. Dongxu, Q. Luo, and K. Liu, “Analysis and testing of microvibrations produced by momentum wheel assemblies," Chinese Journal of
Aeronautics, 2012, 25(4): 640-649.

[30] W. Y. Zhou, G. S. Aglietti, and Z. Zhang, "Modelling and testing of a soft suspension design for a reaction/momentum wheel assembly," Journal of Sound and Vibration, 2011, 330(18): 45964610 . 